\title{
FGFR inhibitor AZD4547 can enhance sensitivity of esophageal squamous cell carcinoma cells with epithelial-mesenchymal transition to gefitinib
}

\author{
HONG LUO $^{1 *}$, JIN QUAN $^{3 *}$, HE XIAO $^{1}$, JIA LUO $^{1}$, QIN ZHANG $^{1}$, GUOCHENG PI $^{1}$, YUNFEI YE ${ }^{1}$, \\ RONG HE $^{1}$, YUN LIU ${ }^{1}$, XIAONA SU ${ }^{1}$, LIANHUA ZHAO ${ }^{2}$ and GE WANG ${ }^{1}$ \\ ${ }^{1}$ Cancer Center and ${ }^{2}$ Department of Pathology, Research Institute of Surgery, Daping Hospital, \\ The Third Military Medical University, Chongqing 400042; ${ }^{3}$ Department of Oncology, \\ The Central Hospital of Jiangjin, Chongqing 402260, P.R. China
}

Received August 30, 2017; Accepted February 15, 2018

DOI: 10.3892/or.2018.6304

\begin{abstract}
Activation of fibroblast growth factor receptor (FGFR) signaling occurs in various cancers, including esophageal squamous cell carcinoma (ESCC), however, the effect of targeting FGFR in ESCC is not clear. Herein, we examined the phosphorylation level of FGFR1 ${ }^{\mathrm{Y} 654}$ (p-FGFR1) in ESCC cell lines and tumor tissues, as well as the cancer cell killing effects of gefitinib and FGFR inhibitor AZD4547 in combination form or alone in ESCC cells. Immunohistochemistry staining was used to detect the expression level of p-FGFR1 in 87 ESCC specimens. The effects of gefitinib and FGFR inhibitor AZD4547 on ESCC cells were analyzed by CCK-8 assay, flow cytometry and western blotting assays. Twenty-six patients diagnosed with esophageal squamous cell carcinoma (ESCC) $(29.9 \%)$ were observed with a high level of p-FGFR1. The proportion of lesions located in the lower segment of the esophagus was significantly higher in the high p-FGFR1 level group (26.9 vs. $8.2 \%, \mathrm{P}=0.003)$. The $\mathrm{IC}_{50}$ values of gefitinib alone and in combination with $500 \mathrm{nM}$ AZD4547 were $22.9 \pm 2.1$ and $4.13 \pm 0.12 \mu \mathrm{M}$ in TE10 cells, and 9.85 \pm 5.5 and $3.21 \pm 0.76 \mu \mathrm{M}$ in EC9706 cells, respectively. The combination of AZD4547 and gefitinib induced robust apoptosis and decreased clone formation ability compared to gefitinib monotherapy in the TE10 cells. TE10 cells exhibited a mesenchymal phenotype, with a higher level of p-FGFR1 and p-AKT than that in EC9706 cells. AZD4547 and gefitinib co-treatment resulted in a significant decrease in the level of p-AKT in
\end{abstract}

Correspondence to: Professor Ge Wang, Cancer Center, Research Institute of Surgery, Daping Hospital, The Third Medical University, 10 Changjiang Zhilu, Yuzhong, Chongqing 400042, P.R. China

E-mail: wangge70@hotmail.com

${ }^{*}$ Contributed equally

Key words: fibroblast growth factor receptor 1, epidermal growth factor receptor, epithelial-mesenchymal transition, esophageal squamous cell carcinoma, gefitinib
TE10 cells and a complete inhibition of phosphorylation of ERK1/2 in EC9706 cells. Collectively, AZD4547 can improve sensitivity of ESCC cells to gefitinib.

\section{Introduction}

Esophageal carcinoma is the sixth most malignant tumor worldwide, and esophageal squamous cell carcinoma (ESCC) is a major pathological subtype in China (1). Despite improvements in comprehensive therapeutics, the prognosis for ESCC remains poor, with a 5-year survival rate of less than $40 \%$ (2). Previous studies have suggested that 30 to $90 \%$ of ESCC patients exhibited a high epidermal growth factor receptor (EGFR) expression level $(3,4)$. However, whether EGFR expression can predict benefits from EGFR TKIs in ESCC is still controversial. Hara et al explored the anticancer effects of gefitinib in ESCC cell lines, and their results suggested that some cell lines with high EGFR level exhibited obvious sensitivity and cell growth inhibition both in vivo and in vitro towards gefitinib (5). However, a phase III multicenter double-blind placebo-controlled randomized trial revealed that the use of gefitinib as a second-line treatment in ESCC in unselected patients did not improve overall survival (6). There was also no clear evidence that patients with ESCC benefit from blocking EGFR with monoclonal antibodies. Altogether, the use of EGFR TKI in ESCC is discouraged at present.

Recently, genomics research of ESCC revealed many copy number variations (CNVs) in genes, such as $S O X 2, P I 3 K C A$, $C C N D 1, F G F R$. The CNVs in these genes may prompt new therapeutic targets for ESCC treatment (7). Fibroblast growth factor receptor (FGFR) pathway activation was revealed to be one of the mechanisms that contribute to resistance to various targeted therapeutics including EGFR TKIs in a variety of solid tumors. Zhang et al reported that a cetuximab-sensitive ESCC xenograft model developed resistance to cetuximab due to FGFR2 gene amplification and overexpression. Inhibition of FGFR2 signaling restored its sensitivity to cetuximab. The antitumor effect may be attributed to inhibition of AKT phosphorylation (8). Although FGFR amplification, mutation, and fusion are the main mechanisms mediating FGFR activation 
and promoting solid tumor development $(9,10)$, tyrosine phosphorylation of FGFR plays a key role in enhancing the activity of the protein. The activation of fibroblast growth factor receptor 1 (FGFR1) can be enhanced by phosphorylation of Y653/654 in the activation loop (11). Thus, inhibiting FGFR phosphorylation would be a promising approach for cancer therapy; and AZD4547, a pan-FGFR inhibitor, can strongly inhibit FGFR phosphorylation and downstream signaling at the cellular level (12).

Epithelial-mesenchymal transition (EMT) is an essential mechanism during development and tissue repair (13). EMT also contributes to primary and acquired resistance to various anticancer drugs. Previous studies have suggested that FGFR signaling pathways can induce EMT in head and neck squamous cell carcinomas, and maintain the EMT phenotype in breast cancer cells $(14,15)$. In the present study, we revealed that p-FGFR1 was predominantly expressed in cells with a mesenchymal phenotype. AZD4547 enhanced the sensitivity of ESCC cells with mesenchymal phenotype to gefitinib. Thus, p-FGFR1 not only serves as an EMT marker, but it can also be used as an efficient biomarker to aptly combine AZD4547 and gefitinib in ESCC treatment.

\section{Materials and methods}

Patients. Patients diagnosed with ESCC who underwent esophagectomy from December 2008 to Novomber 2014 were enrolled. The cohort included a total of 87 cases, with 73 males and 14 females. The median age was 60 . Participants who smoked $>1$ pack/day for $>1$ year were considered smokers. A total of 54 participants were smokers and the rest were non-smokers. The clinical stage and pathological grade of differentiation were based on the eighth edition of the AJCC esophageal cancer staging criteria. The number of patients with poor, moderate, and good differentiation were 21, 36 , and 16, respectively. All patients provided written informed consent for biomarker analysis.

Cell lines and antibodies. Human ESCC cell lines TE10 and EC9706 were purchased from the American Type Culture Collection and cultured in Dulbecco's modified Eagle's medium (HyClone; Thermo Fisher Scientific, Logan, UT, USA) supplemented with $10 \%$ fetal bovine serum (FBS), $100 \mu \mathrm{g} / \mathrm{ml}$ of penicillin, and $100 \mathrm{mg} / \mathrm{ml}$ of streptomycin in a humidified incubator at $37^{\circ} \mathrm{C}$, with $5 \% \mathrm{CO}_{2}$. Exponentially growing cells were trypsinized for subculture or further experiments. CCK-8 kits were purchased from Dojindo Molecular Techologies, Inc. (Kumamoto, Japan). The following primary antibodies were obtained from Cell Signaling Technology (Danvers, MA, USA): p-EGF (Tyr1068) (1:1,000 dilution; cat. no. 3777); p-p44/42 MAPK (Erk1/2) (Thr202/Tyr204) (1:2,000 dilution; cat. no. 4370); p-Akt (Ser473) (1:1,000 dilution; cat. no. 9271); horseradish peroxidase (HRP)-conjugated anti-rabbit (1:5,000 dilution; cat. no. 7074); and HRP-conjugated anti-mouse (1:5,000 dilution; cat. no. 7076). The primary antibody against p-FGFR1Y654 (1:500 dilution; cat. no. ab59194) and $\beta$-actin (1:5,000 dilution; cat. no. ab8226) were purchased from Abcam (Cambridge, UK). TGF- $\beta$ was obtained from Prospect Inc. (Lebanon, TN, USA). Both AZD457 and gefitinib were purchased from AstraZeneca (Cambridge, UK).
Immunohistochemistry. Tissues were fixed overnight in $4 \%$ paraformaldehyde and embedded in paraffin. Sections $(3 \mu \mathrm{M})$ were deparaffinized and hydrated before peroxide blocking with $3 \% \mathrm{H}_{2} \mathrm{O}_{2}$ in methanol at $4^{\circ} \mathrm{C}$ for $20 \mathrm{~min}$. The rabbit polyclonal antibody against p-FGFR1Y654 was applied at a final concentration of $1.5 \mathrm{~g} / \mathrm{ml}$ and incubated for $90 \mathrm{~min}$ at $37^{\circ} \mathrm{C}$. After incubation with the biotin-labeled goat anti-rabbit IgG pre-diluted by the supplier (Biotin-Streptavidin HRP Detection Systems; cat. no. SP-9001; Beijing Zhongshan Golden Bridge Biological Technology, Co., Ltd., Beijing, China) at room temperature for $1 \mathrm{~h}$ and subsequent application of a streptavidin horseradish peroxidase enzyme complex for $30 \mathrm{~min}$, the sections were stained with diaminobenzidine for $15 \mathrm{~min}$. The specimens were evaluated by two skilled microscopists who had no knowledge of the clinical details of the cases. Only clear staining of the cytoplasm and/or nuclei was considered positive. The expression score of p-FGFR1Y654 was represented as the percentage of positive tumor cells irrespective of staining intensities/slide (0-100\%). CCK-8 assay. Exponentially growing TE10 and EC9706 cells were seeded into 96-well plates (3,000 cells/well). After incubation overnight, the cells were treated with gefitinib at concentrations ranging from $0.125-8 \mu \mathrm{M}$ with or without the FGFR inhibitor AZD4547 (500 nM) for 6 days. Cell viability was assessed using the colorimetric readings obtained at $450 \mathrm{~nm}$ after incubation with CCK-8 for $2 \mathrm{~h}$ (Bio-Rad Laboratories, Inc., Hercules, CA, USA). All assays were performed independently at least three times. The $\mathrm{IC}_{50}$ values were calculated using the median-effect equation proposed by Chou-Talalay (16).

Colony formation assays. Cells were treated with gefitinib with or without AZD4547 for $48 \mathrm{~h}$ before being seeded in $60 \mathrm{~mm}$ petri dishes $\left(3 \times 10^{3}\right.$ cells/petri dish). After 14 days, all petri dishes were washed with PBS, fixed with $4 \%$ formaldehyde, and stained with $0.1 \%$ crystal violet. The colonies that contained $>50$ cells were counted.

Gene expression analysis of 26 esophageal carcinoma cell lines. Gene expression data from esophageal carcinoma cell lines were obtained from the CCLE database (CCLE_ Expression_Entrez_2012-09-29.gct; http://www.broadinstitute. org/ccle/home). Unsupervised hierarchical clustering based on eight mesenchymal markers was performed on 26 esophageal carcinoma cell lines using the Cluster 3.0 program in the Biopython package (http://www.biopython.org) (17) based on eight mesenchymal markers and was visualized using JAVA TreeView program (http://jtreeview.sourceforge.net/) (18). Ten cell lines including TE10 were classified as mesenchymal-like cell lines. The Kruskall-Wallis test was used to determine the mRNA expression difference of four subtypes of FGFR and ERBB3 between epithelial and mesenchymal like cell lines.

Quantitative RT-PCR. Total RNA was isolated with TRIzol and cDNA was synthesized using TaqMan reverse transcription reagents. Real-time PCR was performed in duplicate in three independent experiments using SYBR Green PCR Master Mix on the CFX96 ${ }^{\mathrm{TM}}$ Real-Time System (Bio-Rad Laboratories, Inc.). GAPDH was used as an endogenous normalization control. The primers used are listed in Table I. 
Table I. The list of primers used in real-time quantitative PCR.

\begin{tabular}{llll}
\hline Gene & \multicolumn{1}{c}{ Sequence (5'-3') } & Tm & PrimerBankID \\
\hline hGAPDH & 5'-GGAGCGAGATCCCTCCAAAAT & 61.6 & $378404907 \mathrm{c} 1$ \\
hGAPDH & 5'-GGCTGTTGTCATACTTCTCATGG & 60.9 & \\
Vimentin & 5'-AGTCCACTGAGTACCGGAGAC & 62.4 & $240849334 \mathrm{c} 2$ \\
Vimentin & 5'-CATTTCACGCATCTGGCGTTC & 62.5 & \\
E-cadherin & 5'-ATTTTTCCCTCGACACCCGAT & 61.5 & 169790842c2 \\
E-cadherin & 5'-TCCCAGGCGTAGACCAAGA & 61.9 & $4507741 \mathrm{a} 1$ \\
Twist1 & 5'-GTCCGCAGTCTTACGAGGAG & 61.7 & $324072669 \mathrm{c} 1$ \\
Sist1 & 5'-GCTTGAGGGTCTGAATCTTGCT & 62.3 & \\
Slug & 5'-CGAACTGGACACACATACAGTG & 60.9 & 60.9 \\
\hline
\end{tabular}

Tm, melting temperature.

Flow cytometric analysis. Cells were left to adhere overnight before being treated with the indicated drugs for $24 \mathrm{~h}$. Cells were then washed twice with phosphate-buffered saline and suspended in pre-chilled $70 \%$ alcohol for immobilization overnight. After digestion with $100 \mu \mathrm{g} / \mathrm{ml}$ of RNase A at $37^{\circ} \mathrm{C}$ for $30 \mathrm{~min}, 50 \mu \mathrm{g} / \mathrm{ml}$ of propidium iodide was added to the cells for $30 \mathrm{~min}$ in the dark. The fluorescence intensity of each cell was then assessed using the MoFlo ${ }^{\mathrm{TM}}$ XDP cell sorter (Beckman Coulter, Inc., Brea, CA, USA).

Western blotting assays. Cells were lysed in RIPA buffer (Beyotime Institute of Biotechnology, Jiangsu, China; cat. no. 2010ES60) on ice for $10 \mathrm{~min}$. Protein supernatant was isolated from whole cell lysates by centrifugation at $12,000 \mathrm{x} \mathrm{g}$ at $4^{\circ} \mathrm{C}$. A total of $50 \mu \mathrm{g}$ of protein from each sample was separated using 10\% SDS-PAGE and electrically transferred to a PVDF membrane (EMD Millipore, Billerica, MA, USA). Non-specific binding was blocked with $5 \%$ bovine serum albumin (BSA) at $37^{\circ} \mathrm{C}$ before blotting with the indicated antibodies, and subsequent detection via Pierce ${ }^{\mathrm{TM}}$ ECL Western Blotting Substrate (Thermo Fisher Scientific, Rockford, IL, USA; cat. no. 32106). $\beta$-actin was used as the loading control. Signal intensity with background correction was quantified using the Quantity One software (Bio-Rad Laboratories, Inc.).

Statistical analysis. A Chi-square test was used to determine the association between the expression of p-FGFR1 and clinicopathological characteristics. All data are represented as the mean \pm standard deviation. Each experiment was repeated at least three times. Significance was determined using two-tailed unpaired Student's t-tests. One-way ANOVA was used for comparisons between groups in cases of $>2$ groups and Bonferroni correction was performed for multiple comparisons. P-values $<0.05$ were considered to be statistically significant.

\section{Results}

Phosphorylation level of FGFR1 in ESCC cell lines and tissues. To determine if p-FGFR1 is distributed in ESCC, we first examined the phosphorylation level of FGFR1 in ESCC tissue samples from patients and four human ESCC cell lines, namely TE10, Eca109, EC9706 and PTEN-/-(PTEN knockout Eca109). A high level of p-FGFR1 was observed in TE10 cells, and a low expression was observed in the other cell lines (Fig. 1A). In addition, the level of p-FGFR1 in tissue samples was detected by IHC staining in 87 cases with ESCC (Fig. 1B). The 75\% quantile of p-FGFR1 expression level was $30 \%$. The patients with p-FGFR1Y654 expression $>30 \%$ were categorized as the high-expression group which was comprised of 26 cases, and the rest were categorized as the low-expression group containing 61 cases. Among all tested clinicopathological characteristics, the p-FGFR1 expression level was only related to tumor location. The proportion of lesions located in the lower segment of the esophagus was significantly higher in the high-expression group compared to the low-expression group (26.9 vs. 8.2\%, $\mathrm{P}=0.003)$ (Table II).

AZD4547 improves the sensitivity of ESCC cell lines to gefitinib. AZD4547 potently inhibited p-FGFR1 and downstream signaling through FRS2 and PLCg at the cellular level (12). A recent study demonstrated that the FGFR pathway was critical for carcinogenesis of hepatocellular carcinoma, and AZD4547 may be beneficial for the treatment of HCC patients with positive expression of p-FGFR1 (19). To explore whether AZD4547 improved the sensitivity of ESCC cell lines to gefitinib, the TE10 cell line, which exhibited high expression of p-FGFR1 and the EC9706 cell line which exhibited a low expression level of p-FGFR1 were selected for subsequent experiments. These cells were treated with various concentrations of gefitinib alone or in combination with AZD4547 (500 nM) for 6 days. The treatment interval of 6 days was chosen mainly because the values of the inhibitory ratio were more sTable and suiTable for fitting, using the median-effect equation in order to avoid fluctuation of data under usual exposure time of 48 or $72 \mathrm{~h}$. The results revealed that gefitinib alone slightly reduced cell viability. The $\mathrm{IC}_{50}$ values of EC9706 and TE10 were $9.85 \pm 5.5$ and $22.9 \pm 2.1 \mu \mathrm{M}$, respectively. However, when combined with $500 \mathrm{nM}$ AZD4547, the $\mathrm{IC}_{50}$ of gefitinib in EC9706 and TE10 was decreased to 3.21 20.76 
A
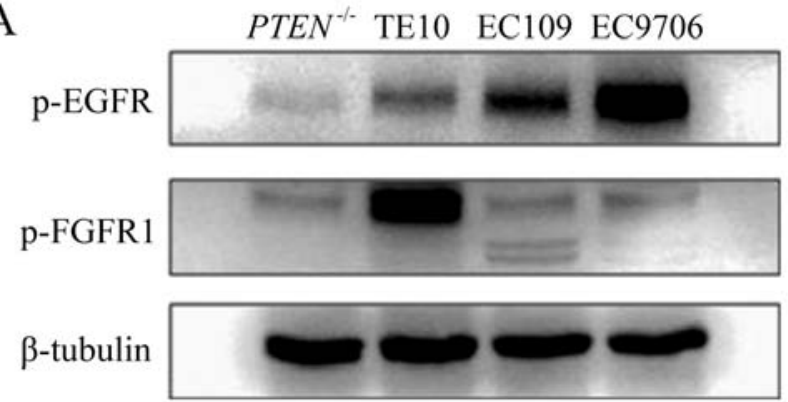

B

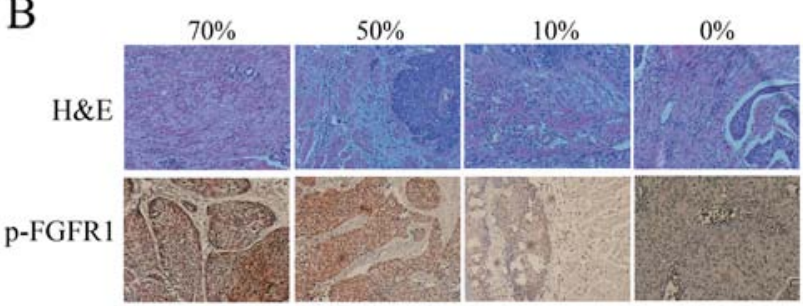

Figure 1. Phosphorylation profile of FGFR1 in ESCC cell lines and tissues. (A) Representative blots of p-FGFR1 and p-EGFR evaluated in four ESCC cell lines. PTEN ${ }^{-/}$denotes ECA109 cells with PTEN knockout. $\beta$-Tubulin was used as a loading control. (B) Representative immunohistochemical images illustrating different levels of p-FGFR1 in tissue samples from ESCC patients. H\&E denotes hematoxylin and eosin staining. FGFR1, fibroblast growth factor receptor 1; ESCC, esophageal squamous cell carcinoma; EGFR, epidermal growth factor receptor.

and $4.13 \pm 0.12 \mu \mathrm{M}$ (Fig. 2A and B), respectively. Moreover, the combination index analysis revealed strong synergism in the range of concentration from 0.125 to $8 \mu \mathrm{M}$ of gefitinib combined with $0.5 \mu \mathrm{M}$ AZD4547 in both of these two cell lines (Fig. 2C). Meanwhile, the combination of AZD4547 and gefitinib led to a marked inhibition of cell survival compared with single-agent gefitinib in colony formation assays $(\mathrm{F}=191.00, \mathrm{P}<0.01$ for $\mathrm{EC} 9706$ in ANOVA; $\mathrm{F}=204.23, \mathrm{P}<0.01$ for TE10 in ANOVA) (Fig. 2D). Collectively, these results indicated that AZD4547 improved the sensitivity of ESCC cell lines to gefitinib. This effect could depend on the phosphorylation status of FGFR1. It is likely that a high level of p-FGFR1 is correlated with greater enhancement of sensitivity to gefitinib.

Effect of AZD4547 and gefitinib combination therapy on the cell cycle and apoptosis in TE10 cells. TE10 cells were divided into four groups: group $\mathrm{C}$ (control), group $\mathrm{G}$ (gefitinib $500 \mathrm{nM}$ ), group A (AZD4547 $500 \mathrm{nM}$ ) and group $A+G$ (gefitinib $500 \mathrm{nM}+A Z D 4547500 \mathrm{nM}$ ). Flow cytometric analysis revealed that gefitinib alone could significantly reduce the proportion of $S$ phase cells $(44.21 \pm 3.96$ vs. $56.40 \pm 4.18$; $\mathrm{P}=0.029)$ and increase the $\mathrm{G} 1$ phase cells $(52.82 \pm 4.08$ vs. $38.87 \pm 3.75 ; \mathrm{P}=0.008)$. Group $\mathrm{A}+\mathrm{G}$ could further significantly reduce $S$ phase cells $(30.66 \pm 4.09$ vs. $44.21 \pm 3.96 ; \mathrm{P}=0.017)$ and promote $\mathrm{G} 1$ phase arrest $(66.71 \pm 3.41$ vs. $2.82 \pm 4.08 ; \mathrm{P}=0.008)$ compared with group $\mathrm{G}$ (Fig. 3A and $\mathrm{B})(\mathrm{F}=23.23, \mathrm{P}<0.01$ for $\mathrm{S}$ phase distribution in the 4 groups; $\mathrm{F}=32.80, \mathrm{P}<0.01$ for G1 phase distribution in the 4 groups). In addition, the combination of AZD4547 and gefitinib induced robust apoptosis compared to gefitinib monotherapy in the TE10 cells revealed by induced upregulation of caspase-9 (Fig. 3C).
Table II. Baseline characteristics of the ESCC patients and associations with p-FGFR1 expression.

\begin{tabular}{|c|c|c|c|}
\hline \multirow[b]{2}{*}{ Factors } & \multicolumn{2}{|c|}{ p-FGFR1 expression } & \multirow[b]{2}{*}{ P-value } \\
\hline & $<30 \%$ & $\geq 30 \%$ & \\
\hline Sex & & & 0.450 \\
\hline Female & $11(18.0)$ & $3(11.5)$ & \\
\hline Male & $50(82.0)$ & $23(88.5)$ & \\
\hline Age (years) & & & 0.251 \\
\hline$<60$ & $27(44.3)$ & $15(57.7)$ & \\
\hline$\geq 60$ & $34(55.7)$ & $11(42.3)$ & \\
\hline Smoking & & & 0.062 \\
\hline Non-smoker & $27(44.3)$ & $6(23.1)$ & \\
\hline Smoker & $34(55.7)$ & $20(76.9)$ & \\
\hline Drinking & & & 0.232 \\
\hline Non-drinker & $32(52.5)$ & $10(38.5)$ & \\
\hline Drinker & $29(47.5)$ & $16(61.5)$ & \\
\hline Anatomical site & & & $0.003^{\mathrm{a}}$ \\
\hline Upper & $9(14.8)$ & $9(34.6)$ & \\
\hline Middle & $47(77.0)$ & $10(38.5)$ & \\
\hline Distal & $5(8.2)$ & $7(26.9)$ & \\
\hline $\mathrm{T}$ & & & $0.665^{\mathrm{a}}$ \\
\hline $\mathrm{T} 2$ & $6(9.8)$ & $3(11.5)$ & \\
\hline $\mathrm{T} 3$ & $40(65.6)$ & $19(73.1)$ & \\
\hline $\mathrm{T} 4$ & $15(24.6)$ & $4(15.4)$ & \\
\hline $\mathrm{N}$ & & & 0.880 \\
\hline No & $15(24.6)$ & $6(23.1)$ & \\
\hline N1-N3 & $46(75.4)$ & $20(76.9)$ & \\
\hline Stage & & & 0.320 \\
\hline I-II & $18(29.5)$ & $5(19.2)$ & \\
\hline III-IV & $43(70.5)$ & $21(80.8)$ & \\
\hline $\begin{array}{l}\text { Complications } \\
\text { after surgery }\end{array}$ & & & 0.446 \\
\hline No & $42(68.9)$ & $20(76.9)$ & \\
\hline Yes & $19(31.1)$ & $6(23.1)$ & \\
\hline Grade & & & $0.782^{\mathrm{a}}$ \\
\hline $\mathrm{Gx}$ & $10(16.4)$ & $4(15.4)$ & \\
\hline G3 & $13(21.3)$ & $8(30.8)$ & \\
\hline $\mathrm{G} 2$ & $27(44.3)$ & $9(34.6)$ & \\
\hline G1 & $11(18.0)$ & $5(19.2)$ & \\
\hline $\begin{array}{l}\text { Serum CEA level } \\
\text { before surgery }\end{array}$ & $0(0.00-4.68)$ & $1.14(0.00-8.05)$ & 0.127 \\
\hline
\end{tabular}

ESCC, esophageal squamous cell carcinoma; p-FGFR1, phosphorylated fibroblast growth factor receptor 1 . ${ }^{a}$ Fisher's exact probability.

Effects of combined AZD4547 and gefitinib on signaling pathways in ESCC cell lines. Koole et al reported that combined AZD4547 and gefitinib resulted in reduced AKT/MAPK signaling in head and neck squamous cell carcinoma (20). In ESCC, the combination of cetuximab and the FGFR inhibitor NVP-BJG398 resulted in a greater inhibition of p-AKT (8). 

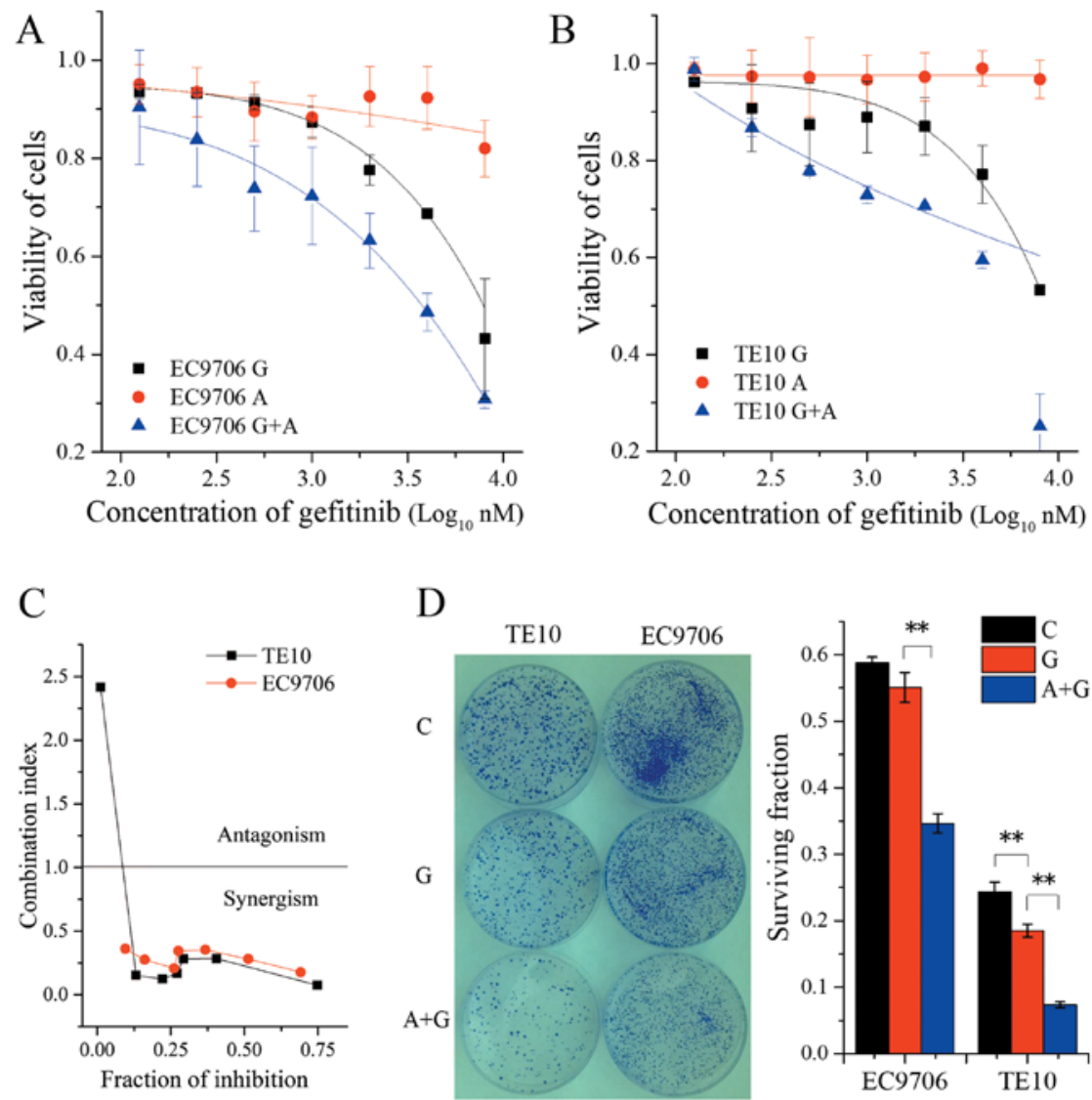

Figure 2. Combination of gefitinib and AZD4547 enhances the inhibitory effect in TE10 and EC9706 cells. (A) Growth inhibitory curves of EC9706 and (B) TE10 cells treated with gefitinib alone or in combination with $500 \mathrm{nM}$ AZD4547. Values are represented as the means \pm SD of three independent experiments. The curves were obtained through four parameters of logistic regression fitted to inhibitory fractions. (C) Quantified data of B. (D) Representative colonies of TE10 and EC9706 cells treated with gefitinib alone or in combination with AZD4547 for $48 \mathrm{~h}$ before clonogenic assay. 'C' denotes control without any treatment; 'G': $2 \mu \mathrm{M}$ gefitinib; and 'A+G': $500 \mathrm{nM}$ AZD4547 plus $2 \mu \mathrm{M}$ gefitinib. ** $\mathrm{P}<0.01$ compared with gefitinib alone.
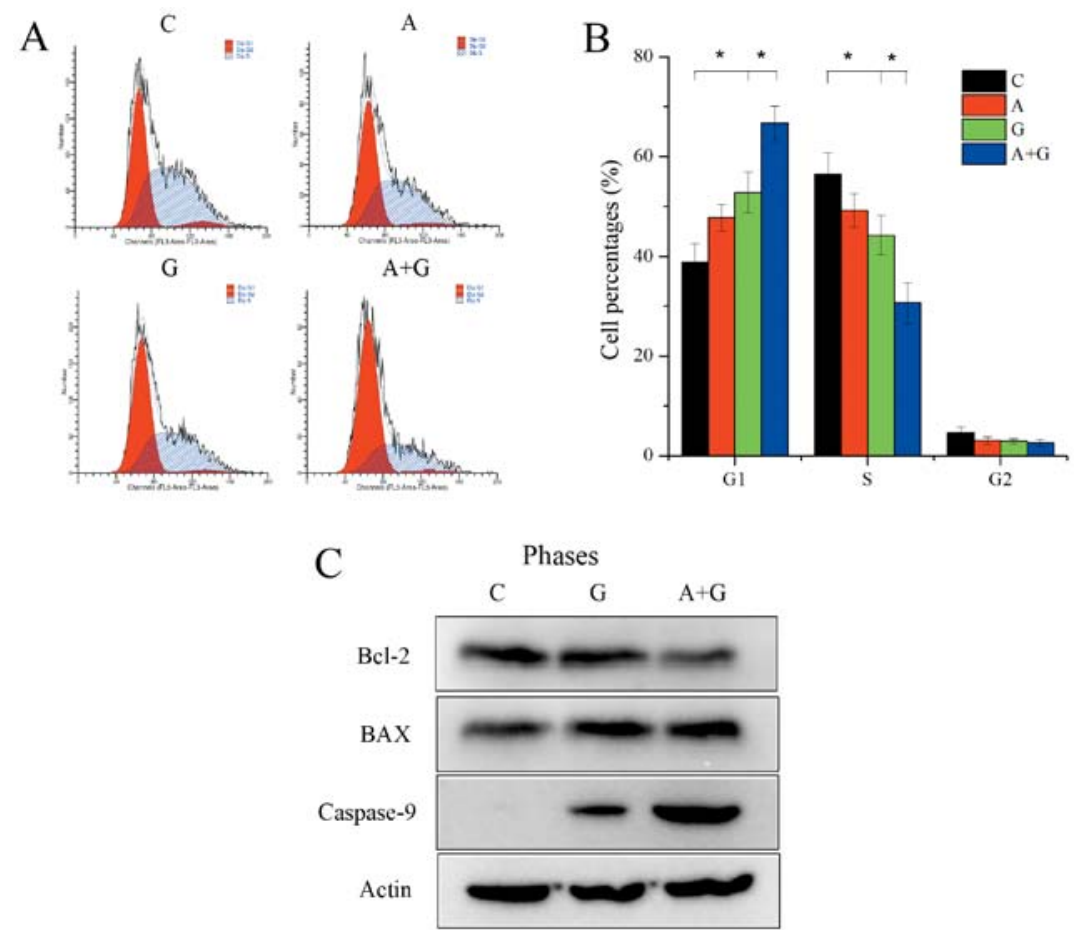

Figure 3. The combination of gefitinib and AZD4547 further enhanced G1 phase arrest and decreased S phase cells compared with gefitinib alone. (A) Representative histograms revealing cell cycle distribution. (B) The quantitative histograms of A. Experiments were performed in triplicate. The percentage of cells in each cell cycle phase is represented as the means \pm SD. " $\mathrm{P}<0.05$ compared with gefitinib alone. (C) Western blotting images revealing the expression level of apoptosis-associated proteins in TE10 cells. 'C' denotes control without any treatment; 'G': $2 \mu$ M gefitinib; 'A': $500 \mathrm{nM}$ AZD4547; and 'A+G': $500 \mathrm{nM}$ AZD4547 plus $2 \mu \mathrm{M}$ gefitinib. 
A

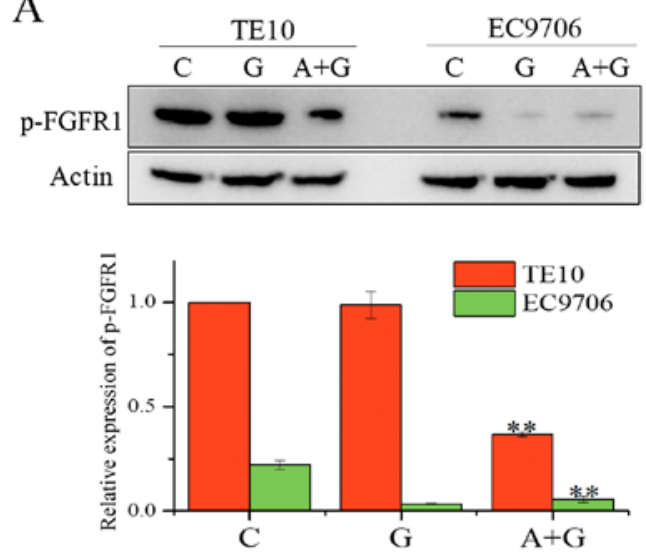

B

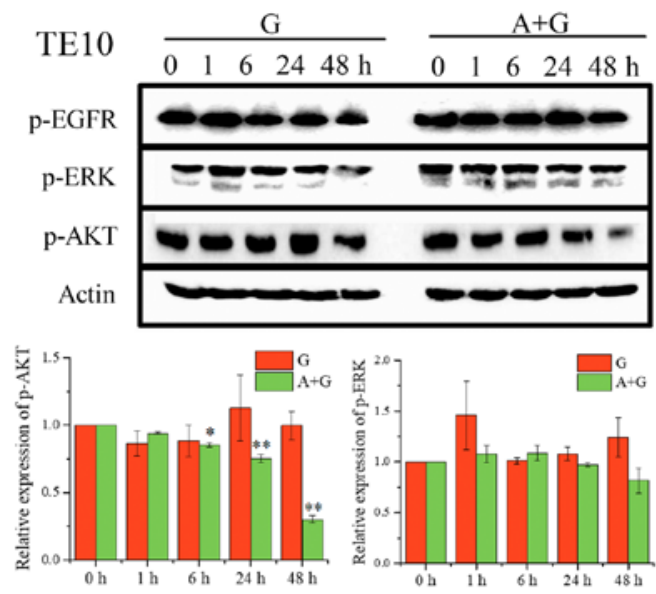

$\mathrm{C}$
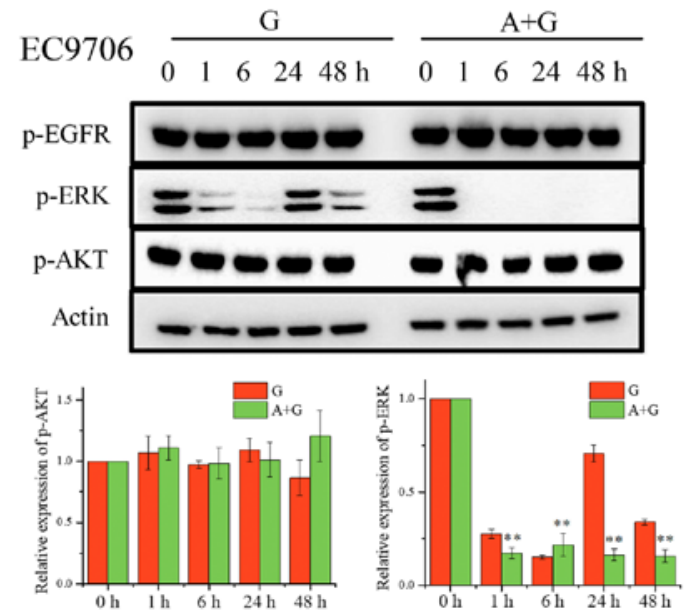

Figure 4. Effects of combined AZD4547 and gefitinib on signaling pathways in ESCC cell lines. (A) Upper panel are the western blots revealing a profound decrease in the expression level of p-FGFR1 when cells were co-treated with AZD4547 and gefitinib. The lower panel is a quantitative histogram of the western blotting results of three independent experiments. ${ }^{* *} \mathrm{P}<0.01$ compared with control. (B) Western blotting images revealing a decrease in the level of p-AKT in TE10 cells co-treated with gefitinib and AZD4547 during the indicated time course. ${ }^{*} \mathrm{P}<0.05,{ }^{* *} \mathrm{P}<0.01$ compared with the 0 h group treated with AZD4547 and gefitinib'. (C) Dual FGFR/EGFR inhibition completely abolished ERK signaling in EC9706 cells. ${ }^{* * *} \mathrm{P}<0.01$ compared with the 0 h group treated with AZD4547 and gefitinib. 'C' denotes control without any treatment; 'G': $2 \mu \mathrm{M}$ gefitinib; 'A': $500 \mathrm{nM}$ AZD4547; and 'A+G': $500 \mathrm{nM}$ AZD4547 plus $2 \mu \mathrm{M}$ gefitinib. ESCC, esophageal squamous cell carcinoma; FGFR1, fibroblast growth factor receptor 1; FGFR, fibroblast growth factor receptor; EGFR, epidermal growth factor receptor.

To assess the effects of the combination of AZD4547 and gefitinib on signaling pathways in ESCC cell lines, we first determined the level of p-FGFR1 in TE10 and EC9706 after treatment with the indicated drugs for $24 \mathrm{~h}$ (Fig. 4A). Western blot analysis revealed that the combination of gefitinib and ZD4547 significantly inhibited the phosphorylation of FGFR1 in TE10 cells. In addition, the level of p-FGFR1 in EC9706 cells was decreased by the combination of AZD4547 and gefitinib. We analyzed the MAPK/AKT pathways in these cell lines (Fig. 4B). The expression of p-AKT in TE10 cells treated with the combination of gefitinib and AZD4547 was gradually inhibited as the treatment duration increased whereas the gefitinib alone treatment group completely failed to inhibit the expression of $\mathrm{p}-\mathrm{AKT}$ ( $\mathrm{F}=563.96, \mathrm{P}<0.01$ for combination; $\mathrm{F}=1.822, \mathrm{P}=0.201$ for gefitinib alone). However, co-treatment did not affect AKT signaling in the EC9706 cells, and dual FGFR/EGFR inhibition completely abolished ERK signaling $(\mathrm{F}=306.82, \mathrm{P}<0.01$ for combination). Collectively, our data revealed that the combination of AZD4547 and gefitinib may affect PI3K/AKT and MAPK/ERK signaling in TE10 cells and EC9706 cells, respectively.

EMT is associated with different signaling pathways for co-treatment in ESCC. Next, to further investigate the reason why co-treatment affects different signaling pathways, we used the CCLE database (CCLE Expression Entrez 2010-09-29. gct; http://www.broadinstitute.org/ccle/home) to analyze 26 ESCC cell lines with 8 genes which were selected from 26 genes that had been used to discriminate between mesenchymal and epithelial phenotype in the previously published study (21). Hierarchical clustering analysis revealed that ESCC cell lines could be classified into two groups based on epithelial and mesenchymal markers, and with TE10 exhibiting a mesenchymal phenotype. Notably, mesenchymal phenotype cells demonstrated significantly higher FGFR1 mRNA expression compared to cells with an epithelial phenotype (Fig. 5A and B). Quantitative RT-PCR ascertained that two critical transcriptional factors, TWIST1 and SLUG that 

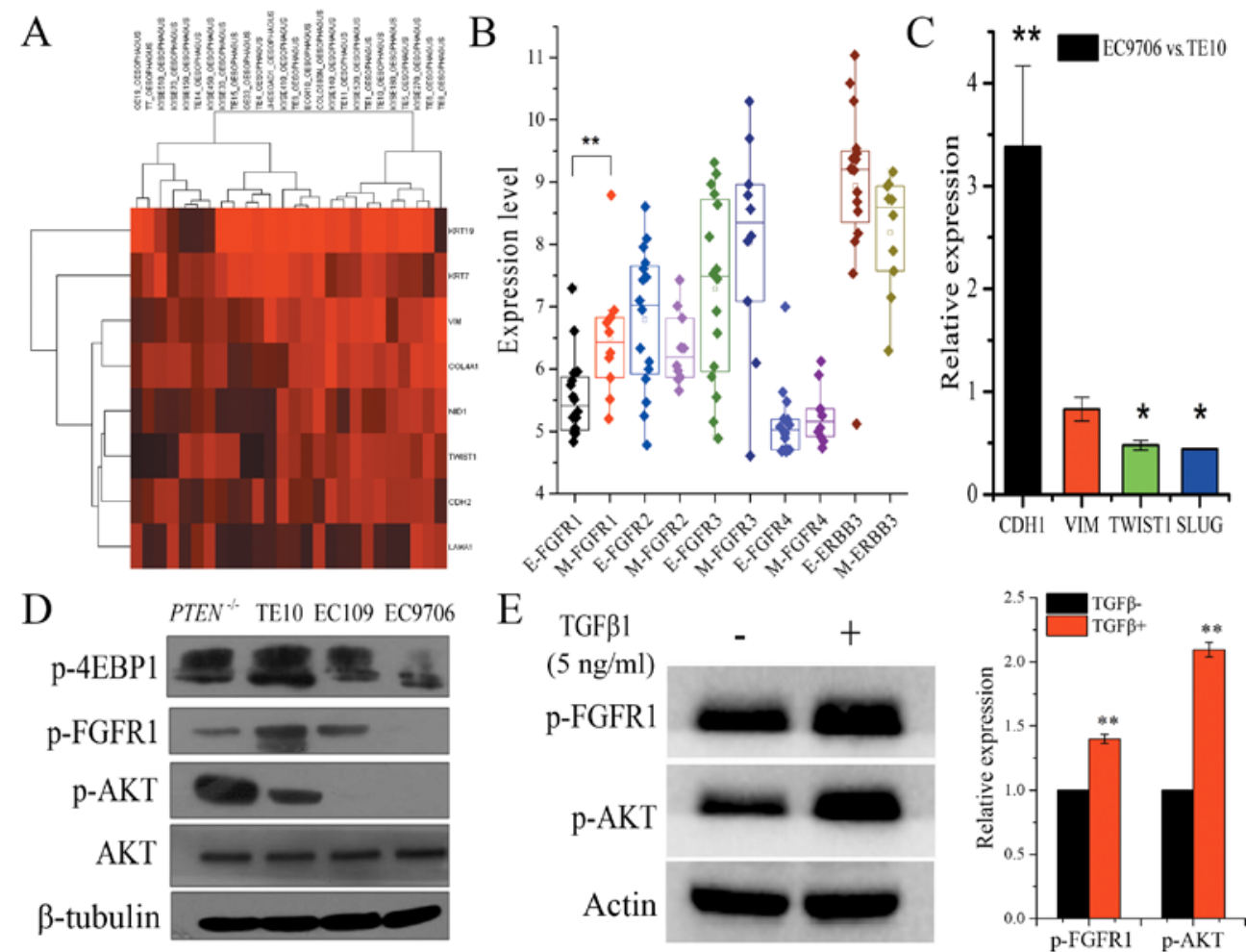

Figure 5. EMT is associated with different signaling pathways in ESCC cell lines. (A) Unsupervised hierarchical clustering of 26 esophageal carcinoma cell lines from the CCLE databases based on eight genes expression. (B) Box plot revealing that only mRNA expression of FGFR1 was significantly higher in cell lines with mesenchymal phenotype. The initial of $\mathrm{E}$ denotes epithelial and $\mathrm{M}$ denotes mesenchymal. (C) Histogram revealing the relative expression level of four EMT-associated genes in EC9706 and TE10 cells determined by real-time quantitative PCR using the $\Delta \Delta \mathrm{Ct}$ method and GAPDH was used as a calibration control. ${ }^{*} \mathrm{P}<0.05,{ }^{* *} \mathrm{P}<0.01$ compared between EC9706 and TE10 cells. (D) Western blots revealing the distinct levels of p-FGFR1 and p-AKT in four ESCC cell lines. PTEN ${ }^{-1}$ denotes ECA109 cells with PTEN knockout. (E) Western blots demonstrating that the level of p-FGFR1 and p-AKT were significantly increased in EC9706 cells induced by $5 \mathrm{ng} / \mathrm{ml}$ TGF- $\beta 1$ for $48 \mathrm{~h} .{ }^{* *} \mathrm{P}<0.01$ compared with cells without TGF- $\beta 1$ treatment by the Student's t-test. Data are represented as the means \pm SD of three independent experiments. EMT, epithelial-mesenchymal transition; ESCC, esophageal squamous cell carcinoma; FGFR1, fibroblast growth factor receptor 1 .

promote the mesenchymal phenotype, exhibited significantly lower expression in EC9706 cells compared to TE10 cells. Additionally, E-cadherin mRNA expression was significantly higher in EC9706 cells compared to TE10 cells (fold change: $3.38 \pm 0.758, \mathrm{P}<0.01$ ), whereas the expression of TWIST1 and SLUG were significantly lower in EC9706 cells (fold change: $0.48 \pm 0.045, \mathrm{P}<0.05 ; 0.44 \pm 0.004, \mathrm{P}<0.05$, respectively), which also argued for the mesenchymal phenotype characteristics of TE10 cells and the epithelial phenotype of EC9706 cells (Fig. 5C). Xuan et al reported that AKT is likely to have a more important role in EMT induced by TGF- $\beta 1$ in EC9706 cells and may contribute to the invasive and metastatic abilities of EC9706 cells (22). We also found a high level of p-AKT in TE10 cells, but it was low in EC9706 cells (Fig. 5D). Meanwhile, the EC109 cells with PTEN deletion exhibited strong upregulated expression of p-AKT (Fig. 5D). Evidence has shown that fractionated IR-mediated EMT in esophageal carcinoma cells is through PTEN-dependent pathways (23). Furthermore, when EC9706 cells were treated with $5 \mathrm{ng} / \mathrm{ml}$ TGF- $\beta 1$ for $48 \mathrm{~h}$, we observed an increased level of p-AKT and p-FGFR1 (Fig. 5E). These data confirmed that cellular EMT status defined the signaling pathways and the effect of co-treatment in ESCC. p-FGFR1 and p-AKT may not only serve as EMT biomarkers, but they can also be used as efficient biomarkers to rationally combine AZD4547 and gefitinib for ESCC treatment.

\section{Discussion}

Aberrant activation of the FGF/FGFR pathway is an important mechanism for the development and progression of a variety of solid tumors. At present, extensive research has shown that FGFR amplification, mutation, and fusion are the main mechanisms of FGFR activation. High copy numbers of FGFR1 correlated with a significantly higher risk of recurrence in patients with ESCC compared to patients with low copy number expansions (10). Overexpression of FGFR2 was associated with tumor growth and patient outcomes in esophagogastric junction adenocarcinoma (24). Notably, the level of phosphorylation plays a key role in enhancing the activity of the FGFRs. The activation of FGFR1 can be enhanced by the phosphorylation of Y653/654 in the activation loop. In the present study, we determined the profile of p-FGFR1 in cells and tissue samples of ESCC (Fig. 1). In clinical practice, gefitinib as well as other EGFR TKIs were still disappointing for the treatment of ESCC characterized with frequent somatic copy number alterations. Therefore, we explored whether AZD4547 could improve the sensitivity of ESCC cell lines to gefitinib. The results revealed that AZD4547 could increase the sensitivity of TE10 and EC9706 cells to gefitinib in different manners. Namely, it appeared that the higher the expression of p-FGFR1 was, the better the effect of enhanced sensitivity that could be achieved (Fig. 2). 
Recently, some studies have shown that FGFR1 plays an important role in mediating and maintaining EMT transition $(14,15,25)$. EMT plays an important role in mechanisms involved in invasion, migration, and resistance to targeted drugs in a wide variety of tumors. Particularly, Xiong et al classified the ESCC patients into four subtypes using a specific 185-gene signature in which tumors marked with mesenchymal phenotype exhibited worse prognosis (21). Conversely, FGFR1 was highly expressed in the mesenchymal-like KRAS-mutant NSCLC, and MEK inhibition relieved feedback suppression of FGFR1, resulting in reactivation of ERK. The combination of the MEK inhibitor and the FGFR inhibitor resulted in tumor shrinkage (26). Consistent with these previous studies, our findings also provided evidence that revealed moderate but significant inhibition of p-AKT under combination treatment of AZD4547 and gefitinib in TE10 cells, which suggested that p-FGFR/AKT activation may improve the growth of TE10 cells featured with mesenchymal phenotype. Conversely, EC9706 cells with an epithelial phenotype were mainly dependent on EGFR/ERK for growth and survival. Taking the present and other evidence into consideration revealing that the FGFR/AKT pathway participated in the EMT process in breast and prostate cancer $(27,28)$, it is likely that TE10 cells relied more on the FGFR/AKT pathway to maintain EMT and survival.

Although AZD4547 enhanced the sensitivity of TE10 cell lines to gefitinib, co-treatment did not completely inhibit p-AKT in TE10 cells. We speculated that more complex biological networks are involved in maintaining EMT in TE10 cells. This hypothesis still warrants further investigation. Furthermore, it is almost a general principle that cancer cells that undergo EMT are resistant to molecular-targeted drugs partially due to dysregulation of apoptosis. In KRAS-mutant NSCLC cell line SW1573 with an EMT phenotype, marked apoptosis cannot be induced with combinational treatment of BGJ398 and trametinib unless the $\mathrm{Bcl}-2 / \mathrm{Bcl}-\mathrm{xL}$ inhibitor ABT263 is added (24). In the present study, although two powerful targeted drugs were utilized, the inhibitory effects on ESCC cells were only moderate. It is therefore necessary to investigate the effect of apoptosis mechanisms on resistance to certain targeted drugs in ESCC cells.

Recently, EGFR amplification has been demonstrated to be a strong predictor for the benefit of gefitinib in patients with esophageal carcinoma (29). However, due to the high complexity of genomic mutations in esophageal carcinoma (7), a single biomarker could be inadequate to predict benefit from gefitinib. Thus, other accompanied molecular alterations may also play important roles. A high level of p-FGFR1 has been considered as one of the important mechanisms in the promotion of EMT. The PI3K/AKT signaling pathway is the key pathway promoting EMT in a variety of solid tumors. The level of p-AKT was significantly increased in EC9706 cells treated with $5 \mathrm{ng} / \mathrm{ml}$ TGF- $\beta$ for $24 \mathrm{~h}$ (22). Based on these lines of evidence, we proposed that the combination of the expression level of p-FGFR1 and p-AKT can serve as the EMT markers in ESCC.

In conclusion, our findings revealed the profile of p-FGFR1 ${ }^{\mathrm{Y} 654}$ and confirmed that sensitization to gefitinib conferred by AZD4547 in ESCC cell lines was dependent on the level of p-FGFR $1^{\text {Y654 }}$. Notably, p-FGFR1 and
p-AKT may serve as EMT markers and efficient biomarkers to rationally combine AZD4547 and gefitinib for the treatment of ESCC.

\section{Acknowledgements}

No special subjects should be acknowledged.

\section{Funding}

No funding was received.

\section{Availability of data and materials}

All authors agreed that materials included in the manuscript, including all relevant raw data, will be made freely available to any researchers who wish to use them for non-commercial purposes, while preserving any necessary confidentiality and anonymity.

\section{Authors' contributions}

HL and JQ made substantial contributions to conception and design. HX, JL, QZ, GP, YY, RH, YL, XS and LZ were all responsible for acquisition of data, or analysis and interpretation of data. GW was involved in drafting the manuscript or revising it critically for important intellectual content. All authors made the final approval of the version to be published.

\section{Ethics approval and consent to participate}

The present study was conducted rigorously according to the Declaration of Helsinki and the principle of International Ethical Guidelines for Biomedical Research Involving Human constituted by the World Health Organization (WHO) and the Council for International Organizations of Medical Sciences (CIOMS). This study was approved by Ethics Committee of the Daping Hospital. Written consent was obtained from all patients for permission of utilization of tissue samples.

\section{Consent for publication}

All the patients have provided written informed consent for the publication of immunohistological images and corresponding statistical data.

\section{Competing interests}

The authors declare that they have no competing interests.

\section{References}

1. Siegel RL, Miller KD and Jemal A: Cancer statistics, 2015. CA Cancer J Clin 65: 5-29, 2015.

2. Ando N, Ozawa S, Kitagawa Y, Shinozawa Y and Kitajima M: Improvement in the results of surgical treatment of advanced squamous esophageal carcinoma during 15 consecutive years. Ann Surg 232: 225-232, 2000.

3. Pande AU, Iyer RV, Rani A, Maddipatla S, Yang GY, Nwogu CE, Black JD, Levea CM and Javle MM: Epidermal growth factor receptor-directed therapy in esophageal cancer. Oncology 73: 281-289, 2007. 
4. Gibault L, Metges JP, Conan-Charlet V, Lozac'h P, Robaszkiewicz M, Bessaguet C, Lagarde N and Volant A: Diffuse EGFR staining is associated with reduced overall survival in locally advanced oesophageal squamous cell cancer. Br J Cancer 93: 107-115, 2005.

5. Hara F, Aoe M, Doihara H, Taira N, Shien T, Takahashi H, Yoshitomi S, Tsukuda K, Toyooka S, Ohta T and Shimizu N: Antitumor effect of gefitinib ('Iressa') on esophageal squamous cell carcinoma cell lines in vitro and in vivo. Cancer Lett 226: 37-47, 2005.

6. Dutton SJ, Ferry DR, Blazeby JM, Abbas H, Dahle-Smith A, Mansoor W, Thompson J, Harrison M, Chatterjee A, Falk S, et al Gefitinib for oesophageal cancer progressing after chemotherapy (COG): A phase 3, multicentre, double-blind, placebo-controlled randomised trial. Lancet Oncol 15: 894-904, 2014.

7. Bandla S, Pennathur A, Luketich JD, Beer DG, Lin L, Bass AJ, Godfrey TE and Litle VR: Comparative genomics of esophageal adenocarcinoma and squamous cell carcinoma. Ann Thorac Surg 93: 1101-1106, 2012.

8. Zhang Y, Pan T, Zhong X and Cheng C: Resistance to cetuximab in EGFR-overexpressing esophageal squamous cell carcinoma xenografts due to FGFR2 amplification and overexpression. J Pharmacol Sci 126: 77-83, 2014.

9. Costa R, Carneiro BA, Taxter T, Tavora FA, Kalyan A, Pai SA, Chae YK and Giles FJ: FGFR3-TACC3 fusion in solid tumors: Mini review. Oncotarget 7: 55924-55938, 2016.

10. Kim HS, Lee SE, Bae YS, Kim DJ, Lee CG, Hur J, Chung H, Park JC, Jung DH, Shin SK, et al: Fibroblast growth factor receptor 1 gene amplification is associated with poor survival in patients with resected esophageal squamous cell carcinoma. Oncotarget 6: 2562-2572, 2015.

11. Furdui CM, Lew ED, Schlessinger $\mathrm{J}$ and Anderson KS: Autophosphorylation of FGFR1 kinase is mediated by a sequential and precisely ordered reaction. Mol Cell 21: 711-717, 2006.

12. Gavine PR, Mooney L, Kilgour E, Thomas AP, Al-Kadhimi K, Beck S, Rooney C, Coleman T, Baker D, Mellor MJ, et al: AZD4547: An orally bioavailable, potent, and selective inhibitor of the fibroblast growth factor receptor tyrosine kinase family. Cancer Res 72: 2045-2056, 2012.

13. Thiery JP, Acloque H, Huang RY and Nieto MA: Epithelial-mesenchymal transitions in development and disease. Cell 139: 871-890, 2009.

14. Brown WS, Akhand SS and Wendt MK: FGFR signaling maintains a drug persistent cell population following epithelial-mesenchymal transition. Oncotarget 7: 83424-83436, 2016.

15. Nguyen PT, Tsunematsu T, Yanagisawa S, Kudo Y, Miyauchi M, Kamata N and Takata T: The FGFR1 inhibitor PD173074 induces mesenchymal-epithelial transition through the transcription factor AP-1. Br J Cancer 109: 2248-2258, 2013.

16. Ashton JC: Drug combination studies and their synergy quantification using the Chou-Talalay method. Cancer Res 1: 2400, 2015.

17. De Hoon MJ, Imoto S, Nolan J and Miyano S: Open source clustering software. Bioinformatics 20: 1453-1454, 2004.
18. Saldanha AJ: Java Treeview-extensible visualization of microarray data. Bioinformatics 20: 3246-3248, 2004.

19. Jo JC, Choi EK, Shin JS, Moon JH, Hong SW, Lee HR, Kim SM, Jung SA, Lee DH, Jung SH, et al: Targeting FGFR pathway in human hepatocellular carcinoma: Expressing pFGFR and pMET for antitumor activity. Mol Cancer Ther 14: 2613-2622, 2015.

20. Koole K, Brunen D, van Kempen PM, Noorlag R, de Bree R, Lieftink C, van Es RJ, Bernards R and Willems SM: FGFR1 is a potential prognostic biomarker and therapeutic target in head and neck squamous cell carcinoma. Clin Cancer Res 22: 3884-3893, 2016.

21. Xiong T, Wang M, Zhao J, Liu Q, Yang C, Luo W, Li X, Yang H, Kristiansen K, Roy B and Zhou Y: An esophageal squamous cell carcinoma classification system that reveals potential targets for therapy. Oncotarget 8: 49851-49860, 2017.

22. Xuan X, Zeng Q, Li Y, Gao Y, Wang F, Zhang H, Wang Z, He H and Li S: Akt-mediated transforming growth factor-b1-induced epithelial-mesenchymal transition in cultured human esophageal squamous cancer cells. Cancer Gene Therapy 21: 238-245, 2014.

23. He E, Pan F, Li G and Li J: Fractionated ionizing radiation promotes epithelial-mesenchymal transition in human esophageal cancer cells through PTEN deficiency-mediated Akt activation. PLoS One 10: e0126149, 2015.

24. Tokunaga R, Imamura Y, Nakamura K, Ishimoto T, Nakagawa S, Miyake K, Nakaji Y, Tsuda Y, Iwatsuki M, Baba Y, et al: Fibroblast growth factor receptor 2 expression, but not its genetic amplification, is associated with tumor growth and worse survival in esophagogastric junction adenocarcinoma. Oncotarget 6: 19748-19761, 2016

25. Brown WS, Tan L, Smith A, Gray NS and Wendt MK: Covalent targeting of fibroblast growth factor receptor inhibits metastatic breast cancer. Mol Cancer Ther 15: 2096-2106, 2016.

26. Kitai H, Ebi H, Tomida S, Floros KV, Kotani $H$, Adachi Y, Oizumi S, Nishimura M, Faber AC and Yano S: Epithelial-to-mesenchymal transition defines feedback activation of receptor tyrosine kinase signaling induced by MEK inhibition in KRAS-mutant lung cancer. Cancer Discov 6: 754-769, 2016.

27. Qian X, Anzovino A, Kim S, Suyama K, Yao J, Hulit J, Agiostratidou G, Chandiramani N, McDaid HM, Nagi C, et al: $\mathrm{N}$-cadherin/FGFR promotes metastasis through epithelial-to-mesenchymal transition and stem/progenitor cell-like properties. Oncogene 33: 3411-3421, 2014.

28. Terry S, El-Sayed IY, Destouches D, Maillé P, Nicolaiew N, Ploussard G, Semprez F, Pimpie C, Beltran H, Londono-Vallejo A, et al: CRIPTO overexpression promotes mesenchymal differentiation in prostate carcinoma cells through parallel regulation of AKT and FGFR activities. Oncotarget 6: 11994-2008, 2015,

29. Petty RD, Dahle-Smith A, Stevenson DAJ, Osborne A, Massie D, Clark C, Murray GI, Dutton SJ, Roberts C, Chong IY, et al: Gefitinib and EGFR gene copy number aberrations in esophageal cancer. J Clin Oncol 35: 2279-2287, 2017. 